\title{
Levels of serum IL6, TNF- $\alpha$ and sLAG3 are changed and correlated with clinical characteristics in Parkinson's disease patients: A case-control study
}

\author{
${ }^{* 1}$ Yuting Zhu $M D,{ }^{* 1}$ Xiaoming Guo $M D,{ }^{1}$ Yong Zhou $M D,{ }^{2}$ Dongmei Zhang $P h D,{ }^{1}$ Xiaoyi \\ Yi $M D,{ }^{1}$ Han Wang $M D,{ }^{3} \mathrm{Li}$ Liu, ${ }^{1}$ Xiangyang Zhu $M D$ \\ *YT Zhu and XM Guo contributed equally and are co-first authors
}

${ }^{1}$ Department of Neurology, ${ }^{2}$ Clinical Medicine Research Center, Affiliated Hospital No. 2 of Nantong University, Nantong; ${ }^{3}$ Northern Jiangsu People's Hospital, Affiliated Hospital to Yangzhou University, Yangzhou, China.

\begin{abstract}
Objective: An increasing body of studies have proved that inflammation plays a crucial role in Parkinson's disease (PD) pathogenesis. IL-6, TNF- $\alpha$ and sLAG3 are associated with immune disorder. This study aimed to examine the expression of serum IL-6, TNF- $\alpha$ and sLAG3 in PD patients from China. Methods: Forty six PD patients and 42 age and gender-matched healthy controls (HC) were recruited. clinical data, disease assessment scale and serum IL-6, TNF- $\alpha$ and sLAG3 were assessed in PD patients. The levels of inflammatory factors were determined by enzyme-linked immunosorbent assay (ELISA). Results: The levels of serum IL-6 and TNF- $\alpha$ were significantly higher in PD patients compared to HC. Serum IL-6 and TNF- $\alpha$ were independent risk factors for PD. The levels of serum SLAG3 and TNF- $\alpha$ were higher in female patients than those of male patients. Significant positive correlation was found between serum TNF- $\alpha$ and H-Y, UPDRS III and HAMA in PD patients. Serum sLAG3 positively correlated with HAMA. Conclusion: Changes in serum inflammatory factors were observed in PD patients, which were correlated with the clinical characteristics. The study supports the previous hypothesis that inflammation is involved in the pathogenesis of PD.
\end{abstract}

Keywords: Parkinson's disease, interleukin (IL)-6, tumor necrosis factor (TNF)- $\alpha$, soluble lymphocyte activation gene-3, inflammation

\section{INTRODUCTION}

Parkinson's disease (PD) is a common agerelated movement disorder. It is characterized by the accumulation of $\alpha$-synuclein ( $\alpha$-syn) and degeneration of dopaminergic neurons in substantia nigra $(\mathrm{SN}) .{ }^{1}$ Although the pathogenesis of PD is unknown, inflammation as well as immune activation play crucial roles in $\mathrm{PD}$ pathogenesis., ${ }^{2,3}$

In fact, neuroinflammation characterized by activation of microglia and astrocytes is a protective mechanism of the central nervous system (CNS) against infection and injury. However, uncontrolled inflammation can lead to excessive damage to cells and tissues. Overproducing inflammatory factors like interleukin (IL)-2, interleukin (IL)-6, tumor necrosis factor (TNF)- $\alpha$ disrupt the blood-brain barrier (BBB) and cause irreversible damage to dopamine neurons. ${ }^{4}$ Changes in the serum and cerebral inflammatory factors were observed in mice following MPTP injection. ${ }^{5}$ Previous case-control studies found that serum inflammatory factors were higher compared to healthy controls (HC) ${ }^{6,7} \mathrm{~A}$ retrospective cohort study based on the entire Taiwan population showed that the incidence of PD in patients with rheumatism was 30 times higher than that in patients without rheumatism. Recently, two nationwide cohort studies have found that Inflammatory bowel disease is related to the risk of Parkinson's disease.$^{8-10}$ Epidemiological studies also found that routine use of nonsteroidal antiinflammatory drugs (NSAIDS) may reduce the risk of PD. ${ }^{11}$ The above results strongly suggest that inflammation is closely related to PD.Among numerous inflammatory factors, IL- 6 and TNF- $\alpha$ 
are two classic inflammatory factors that have extensively studied, though these results were contradicted. $6,7,12,13$

Lymphocyte activation gene-3 (LAG3) gene locates in chromosome 12p13, encoding a transmembrane protein homologous with $\mathrm{CD} 4$, which has function as ligand of major histocompatibility complex (MHC) class II competing with $\mathrm{CD} 4+\mathrm{T}$ cells in regulating immune system. ${ }^{14}$ Recent studies have shown LAG3 is related to the spread of $\alpha$-syn and neuroinflammation in PD. ${ }^{15}$ It is worth mentioning that mature LAG3 can fell out from the cell membrane to form soluble LAG3 (sLAG3), which can be measured in serum. ${ }^{14}$ Whereas, there are few studies on the expression of serum sLAG3 in PD patients.

These three inflammatory factors were closely connected to the pathology of PD. Thus, to further address the possible relationship between inflammation and PD, we conducted a case-control study to examine the expression of serum IL-6, TNF- $\alpha$ and sLAG3 in PD patients from China.

\section{METHODS}

\section{Study participants}

A total of 88 participants were enrolled in this study (46 PD patients and 42 HC) from the Department of Neurology of the Second Affiliated Hospital of Nantong University between February 2019 to October 2019. Patients met the clinical diagnosis of PD according to the EFNS and MDSES $^{16}$ recommendations. Participants who suffer from autoimmune diseases, tumors, infections, severe dysfunction of the liver and the kidney and other neurodegenerative diseases and take low-inflammatory drugs were excluded. The study was approved by the Second Affiliated Hospital of Nantong University ethics committee and all participants signed informed consent.

\section{Clinical data and disease assessment scales}

Baseline data including age, gender were obtained in two groups. Age of onset, disease duration were obtained in PD patients. The severity of motor symptoms was assessed by Hoehn and Yahr scale (H-Y) stage, Unified Parkinson's Disease Rating Scale (UPDRS) Part III. PD patients can be grouped into tremor-dominant (TD), postural instability and gait disorder (PIGD) and intermediate (I) according to the predominant motor symptoms. ${ }^{17}$ The motor examinations were done in the OFF state. Non-motor like emotional disorder was assessed using Hamilton
Depression Scale (HAMA), Hamilton Anxiety Scale (HAMD).

\section{Sample collection and measurement}

Blood samples were gathered from patients between 6:00 a.m. and 7:00 a.m. after overnight fasting. Venous blood from participants was centrifuged at a speed of $2500 \mathrm{~g}$ force for 20 min to obtain serum sample. Serum sLAG-3 was measured by human sLAG-3 Enzymelinked immunosorbent assay (ELISA) (Abcam, Cambridge, UK) kit. Serum IL-6 and TNF- $\alpha$ were estimated using commercially available human IL-6 ELISA kit (catalog Number: EEL-H0102), human TNF- $\alpha$ ELISA kit (catalog Number: E-EL-H0109).

\section{Statistical analysis}

Age, age of onset, serum IL-6, TNF- $\alpha$ and sLAG3 were expressed as mean \pm SD and compared by $\mathrm{t}$-test. H-Y and disease duration were expressed as median (IQR). Enumeration data was presented as percentage and was compared by $\chi 2$ test. Multivariate binary logistic regression analyses were performed to determine independent correlation factors for PD. Subgroup analysis and spear-man correlation coefficient were performed to explore the relationship between inflammatory factors and clinical characteristics. $\mathrm{P}<0.05$ was considered significantly significant.

\section{RESULTS}

\section{Characteristics of participants}

Table 1 showed the clinical features of PD patients $(n=46)$ and $\mathrm{HC}(\mathrm{n}=42)$. There were no differences in sex and age $(\mathrm{P}>0.05)$. The $\mathrm{PD}$ patients were made up 18 females and 28 males with mean age of $69.48 \pm 9.60$ years. The HC composed of 18 females and 28 males with mean age of $69.71 \pm$ 9.04 years.

Levels of serum IL-6, TNF- $\alpha$, sLAG3 in two groups

As shown in Table 1, the levels of serum IL-6, TNF- $\alpha$ were significantly higher $(\mathrm{P}=0.003 ; \mathrm{P}=$ 0.001 ) in PD patients than those in HC. However, no significant difference was found in the levels of serum sLAG3 between two groups (Table 1). Based on bivariate logistic regression analysis, serum IL6 (odds ratio, OR 1.078, 95\% confidence interval, CI 1.004-1.156, $\mathrm{P}=0.038)$ and TNF- $\alpha$ (OR 1.144, 95\% CI $1.026-1.276, \mathrm{P}=0.015)$ were independent risk factors for PD (Table 2). 
Table 1: Demographic and clinical features of PD patients and HC

\begin{tabular}{|c|c|c|c|c|}
\hline Item & $\begin{array}{c}\text { PD patients } \\
n=46\end{array}$ & $\begin{array}{c}\mathrm{HC} \\
\mathrm{n}=42\end{array}$ & $t / \chi^{2}$ & $\mathbf{P}$ \\
\hline Age, $($ mean \pm SD) & $69.48 \pm 9.60$ & $69.71 \pm 9.04$ & -0.119 & 0.906 \\
\hline Gender, (female/male) & $18 / 28$ & $18 / 24$ & 0.722 & 0.126 \\
\hline Age of onset, $($ mean $\pm S D)$ & $65.11 \pm 8.99$ & - & - & - \\
\hline Disease duration, median (IQR) & $3(1,8)$ & - & - & - \\
\hline H-Y stage, $($ mean \pm SD) & $2(2,3)$ & - & - & - \\
\hline UPDRS III, (mean \pm SD) & $26.39 \pm 12.63$ & - & - & - \\
\hline HAMA, (mean \pm SD) & $11.22 \pm 5.35$ & - & - & - \\
\hline HAMD, (mean \pm SD) & $10.17 \pm 4.83$ & - & - & - \\
\hline sLAG3, (mean \pm SD) & $119.54 \pm 52.17$ & $143.46 \pm 90.35$ & -1.503 & 0.138 \\
\hline IL6, (mean \pm SD) & $15.35 \pm 8.14$ & $10.76 \pm 5.79$ & 3.067 & $0.003 * *$ \\
\hline $\mathrm{TNF}-\alpha,($ mean $\pm \mathrm{SD})$ & $10.15 \pm 5.75$ & $6.65 \pm 3.60$ & 3.456 & $0.001 * *$ \\
\hline
\end{tabular}

Abbreviations: PD, Parkinson's disease; SD, standard deviation; IQR, interquartile range; H-Y, Hoehn and Yahr; UPDRS III, Unified Parkinson's Disease Rating Scale Part III; HAMA, Hamilton Depression Scale, HAMD, Hamilton Anxiety Scale; sLAG3, soluble lymphocyte activation gene-3; IL6, interleukin-6; TNF- $\alpha$, tumor necrosis factor $-\alpha$; $* \mathrm{P}<0.05$, $* * \mathrm{P}<0.01$.

Comparison of serum IL-6, TNF- $\alpha$, sLAG3 in PD patients with different subgroups

The serum levels of TNF- $\alpha$ and sLAG3 were higher $(\mathrm{P}=0.028 ; \mathrm{P}=0.037)$ in female patients when compared to male patients. No statistical differences were observed in age and motor subtypes (Table 3).

Correlations between serum IL-6, TNF- $\alpha$, sLAG3 and clinical characteristics of $P D$ patients

The Spear-man correlation analyses showed that TNF- $\alpha$ positively correlated with $\mathrm{H}-\mathrm{Y}(\mathrm{r}=0.370$, $\mathrm{P}=0.011)$ (Figure 1A), UPDRS III $(\mathrm{r}=0.320, \mathrm{P}=$ 0.03 ) (Figure 1B), and HAMA $(r=0.301, P=0.042)$ (Figure 1C), in PD patients. Furthermore, serum sLAG3 positively correlated with $\operatorname{HAMA}(\mathrm{r}=$ $0.393, \mathrm{P}=0.007$ ) (Figure 1D). No significant correlation were found between inflammatory factors and other clinical features.

\section{DISCUSSION}

Recent studies have found that inflammation is involved in the pathogenesis of neurodegenerative diseases including PD. In PD patients, excessive inflammatory factors could enter the circulation through the damaged $\mathrm{BBB}$, causing the raised serum levels of inflammatory factors. Consistent with our assumption, the serum levels of IL-6 and TNF- $\alpha$ in PD patients increased compared with HC. And elevated serum IL6 and TNF- $\alpha$ were independent risk factors for PD.

Aggregation of $\alpha$-syn in $\mathrm{SN}$ is a classic pathological feature of PD[1]. In 2003, Braak proposed that the pathological changes of PD started from the anterior olfactory nucleus and the vagus nerve and then developed upward, gradually involved the brain stem, substantia nigra, limbic system, and cortex, which often related to the development of motor and non-motor symptoms. ${ }^{18,19}$ Mao et al. found LAG3 can mediate endocytosis and cell-to-cell transmission of $\alpha$-syn by high affinity to $\alpha$-syn preformed fibrils (PFFs). ${ }^{15}$ Thus, it is reasonable to speculate that its shed and detectable sLAG3 may reflect the severity of PD. However, no significant difference was found

Table 2: Multivariate binary logistic regression analysis of risk factors for PD

\begin{tabular}{lcccccc}
\hline & B & SE & Wald & P & OR & 95\% CI \\
\hline IL-6 & 0.075 & 0.036 & 4.3 & $0.038^{*}$ & 1.078 & $1.004-1.156$ \\
TNF- $\alpha$ & 0.135 & 0.055 & 5.907 & $0.015^{*}$ & 1.144 & $1.026-1.276$ \\
\hline
\end{tabular}

Abbreviations: PD, Parkinson's disease; SE, standard error; OR, odd ratio; CI, confidence interval; IL6, interleukin-6; TNF- $\alpha$, tumor necrosis factor $-\alpha ; * \mathrm{P}<0.05, * * \mathrm{P}<0.01$. 
Table 3: Subgroup analysis of serum IL-6, TNF- $\alpha$, sLAG3 in PD patients

\begin{tabular}{|c|c|c|c|}
\hline Variate & IL6 & TNF- $\alpha$ & SLAG3 \\
\hline \multicolumn{4}{|l|}{ Age } \\
\hline$\leqslant 70(25)$ & $16.60 \pm 8.70$ & $9.76 \pm 5.59$ & $116.43 \pm 53.28$ \\
\hline$>70(21)$ & $13.87 \pm 7.35$ & $10.62 \pm 6.04$ & $123.24 \pm 51.89$ \\
\hline $\mathrm{t}$ & -1.14 & 0.499 & 0.436 \\
\hline $\mathrm{p}$ & 0.260 & 0.620 & 0.665 \\
\hline \multicolumn{4}{|l|}{ Gender } \\
\hline Female (18) & $17.64 \pm 9.31$ & $12.45 \pm 6.25$ & $139.42 \pm 54.44$ \\
\hline Male (28) & $13.88 \pm 7.08$ & $8.67 \pm 4.97$ & $106.76 \pm 47.31$ \\
\hline $\mathrm{t}$ & -1.554 & -2.279 & -2.155 \\
\hline $\mathrm{p}$ & 0.127 & $0.028 *$ & $0.037 *$ \\
\hline \multicolumn{4}{|c|}{ Motor sub-types } \\
\hline TD (18) & $12.36 \pm 7.57$ & $7.72 \pm 4.27$ & $120.56 \pm 58.95$ \\
\hline I (15) & $19.24 \pm 9.25$ & $11.08 \pm 5.61$ & $113.82 \pm 51.82$ \\
\hline PIGD (13) & $15.02 \pm 5.91$ & $12.43 \pm 6.78$ & $124.73 \pm 45.61$ \\
\hline $\mathrm{F}$ & 3.233 & 3.088 & 0.152 \\
\hline $\mathrm{p}$ & 0.050 & 0.056 & 0.859 \\
\hline
\end{tabular}

Abbreviations: PD, Parkinson's disease; TD, tremor-dominant; PIGD, postural instability and gait disorder; I, intermediate; sLAG3, soluble lymphocyte activation gene-3; IL6, interleukin-6; TNF- $\alpha$, tumor necrosis factor $-\alpha$; $* \mathrm{P}<0.05$, ** $\mathrm{P}<0.01$.
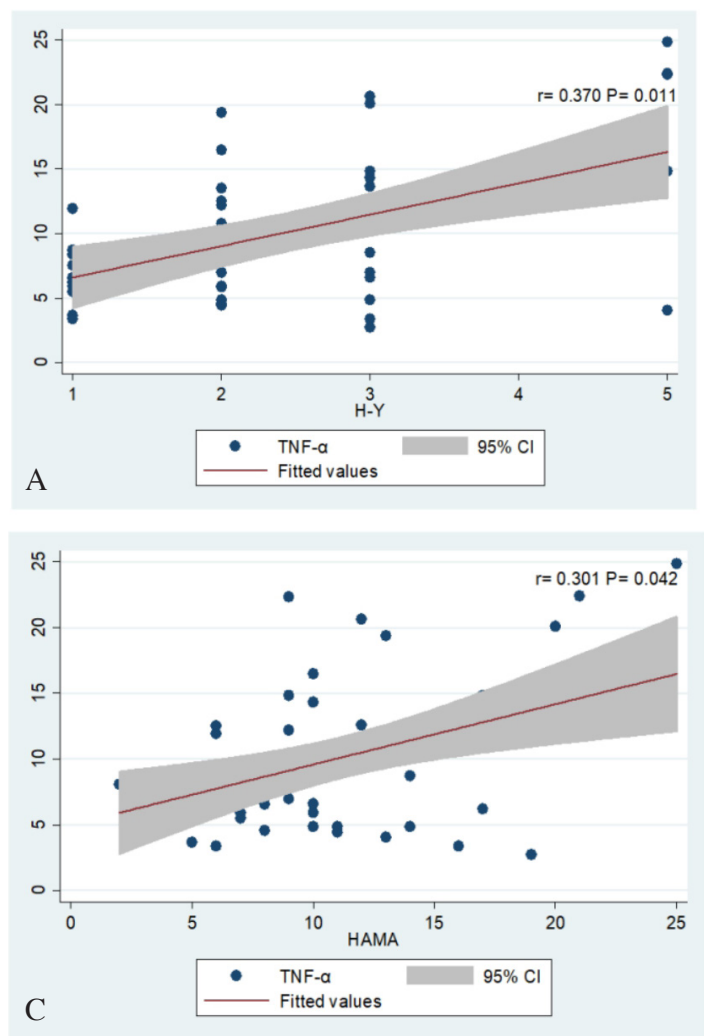

Figure 1A-D. The Spear-man correlation analyses.

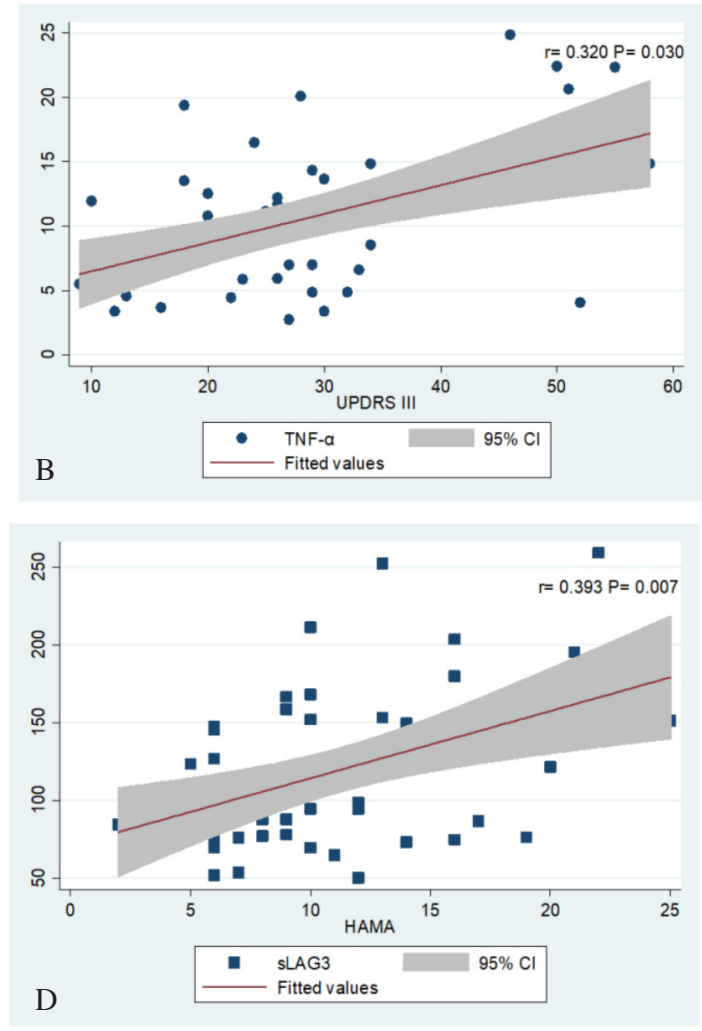


in the level of sLAG3 between two groups. The result was inconsistent with a previous study ${ }^{20}$, which showed that PD patients had significantly higher levels of sLAG3. The reason why there was no statistical difference could be attributed to our small sample size. In addition, We could not determine whether serum sLAG3 entirely represents intracranial LAG3 levels. Interestingly, in PD subgroup analysis, we found that serum sLAG3 levels were significantly higher in women than those in men. Epidemiological evidence of gender differences in PD suggests that estradiol in circulation in women may have beneficial effects on the dopaminergic system, acting as a neuroprotective agent by inhibiting inflammation. Women with a higher exposure to estrogen have a significantly lower risk of developing PD. ${ }^{21}$ Postmenopausal women are more prone to immune disorders due to the decreased estrogen and more active inflammation. ${ }^{22}$ Therefore, higher sLAG3 in women may represent a more active inflammatory response.

TNF- $\alpha$, produced by macrophages, lymphoid cells and neurons, plays an important role in activating the transcription factors of nuclear factor kappa-B (NF- $\mathrm{B}$ ) signaling pathway to enhance the inflammation. ${ }^{23}$ Ebrahim Kouchaki et $a l$. found that there was a significant correlation between serum levels of TNF- $\alpha$ and H-Y. ${ }^{6}$ Forbes MP et al. suggested that inflammatory factors are involved with emotional disorders. ${ }^{24}$ Wang et al. showed that TNF- $\alpha$ was associated with severity of anxiety in PD patients. ${ }^{25}$ Consistent with these previous studies, we found that TNF- $\alpha$ was positively correlated with H-Y, UPDRS III and HAMA.

There are some limitations in this study. Firstly, sample size was relatively small. Secondly, the association between inflammatory factors and clinical characteristics in CSF, was not explored.

In conclusion, changes in serum inflammatory factors were observed in PD patients, which were correlated with the clinical characteristics. Though the underlying mechanism is unclear, we could speculate that peripheral inflammatory disorders may in some degree reflect inflammation in brain.

\section{DISCLOSURE}

Financial support: The study was supported by Nantong Science and Technology Project (MS12015093, MS12018042, JC2019031), Foundation of Health Commission of Jiangsu Province (H2018035, H2019057).

Conflict of interest: None

\section{REFERENCES}

1. Kalia LV, Lang AE. Parkinson's disease. Lancet 2015; 386(9996): 896-912.

2. Hirsch EC, D.G. Standaert DG. Ten unsolved questions about neuroinflammation in Parkinson's disease. Mov Disord 2021; 36(1):16-24.

3. Kline EM, Houser MC, Hervick MK, et al. Genetic and environmental factors in Parkinson's disease converge on immune function and inflammation. Mov Disord 2021; 36(1): 25-36.

4. Haruwaka K, Ikegani A, Tachibana Y, et al. Dual microglia effects on blood brain barrier permeability induced by systemic inflammation. Nat Commun 2019; 10(1): 5816.

5. Manocha GD, Floden AM, Puig KL, NagamotoCombs K, Scherzer CR, Comb CK. Defining the contribution of neuroinflammation to Parkinson's disease in humanized immune system mice. Mol Neurodegener 2017; 12(1): 17.

6. Kouchaki E, Kakhaki RD, Tamtaji OR, et al. Increased serum levels of TNF- $\alpha$ and decreased serum levels of IL-27 in patients with Parkinson disease and their correlation with disease severity. Clin Neurol Neurosurg 2018; 166: 76-9.

7. Gupta V, Garg RK, Khattri S. Levels of IL-8 and TNF- $\alpha$ decrease in Parkinson's disease. Neurol Res 2016; 38(2): 98-102.

8. Chang CC, Lin TM, Chang YS, et al. Autoimmune rheumatic diseases and the risk of Parkinson disease: a nationwide population-based cohort study in Taiwan. Ann Med 2018; 50(1): 83-90.

9. Villumsen M,Aznar S, Pakkenberg B, Jess T, Brudek T. Inflammatory bowel disease increases the risk of Parkinson's disease: a Danish nationwide cohort study 1977-2014. Gut 2019 68(1): 18-24.

10. Weimers $\mathrm{P}$, Halfvarson $\mathrm{J}$, Sachs $\mathrm{MC}$, et al. Inflammatory bowel disease and Parkinson's disease: A nationwide Swedish cohort study. Inflamm Bowel Dis 2019; 25(1): 111-23.

11. San Luciano M, Tanner CM, Meng C, et al. Nonsteroidal anti-inflammatory use and LRRK2 Parkinson's disease penetrance. Mov Disord 2020; 35(10): 1755-64.

12. Scalzo P, Kümmer A, Cardoso F, Teixeira AL. Increased serum levels of soluble tumor necrosis factor-alpha receptor-1 in patients with Parkinson's disease. J Neuroimmunol 2009; 216(1-2): 122-5.

13. Chen H, O'Reilly EJ, Schwarzschild MA, Ascherio A. Peripheral inflammatory biomarkers and risk of Parkinson's disease. Am J Epidemiol 2008; 167(1): 90-5.

14. Graydon CG, Mohideen S, Fowke KR. LAG3's enigmatic mechanism of action. Front Immunol 2020; 11: 615317.

15. Mao XB, Ou MTH, Karuppagounder SS, et al. Pathological $\alpha$-synuclein transmission initiated by binding lymphocyte-activation gene 3 . Science 2016; 353(6307): aah3374.

16. Berardelli A, Wenning GK, Antonini A, et al. EFNS/ MDS-ES/ENS [corrected] recommendations for the diagnosis of Parkinson's disease. Eur J Neurol 2013; 20(1):16-34. 
17. Huertas I, Jesús S, Lojo JA, et al. Lower levels of uric acid and striatal dopamine in non-tremor dominant Parkinson's disease subtype. PLoS One 2017; 12(3): e0174644.

18. Braak H, Tredici KD, Rüb U, de Vos RAI, Jansen Steur ENH, Braak E. Staging of brain pathology related to sporadic Parkinson's disease. Neurobiol Aging 2003; 24(2):197-211.

19. Lubomski M, Tan AH, Lim SY, Holmes AJ, Davis RL, Sue CM. Parkinson's disease and the gastrointestinal microbiome. J Neurol 2020; 267(9): 2507-23.

20. Cui SS, Du JJ, Liu SH, et al. Serum soluble lymphocyte activation gene-3 as a diagnostic biomarker in Parkinson's disease: A pilot multicenter study. Mov Disord 2019; 34(1): 138-41.

21. Cerri S, Mus L, Blandini F. Parkinson's disease in women and men: What's the difference? J Parkinsons Dis 2019; 9(3): 501-15.

22. Berchtold NC, Cribbs DH, Coleman PD, et al. Gene expression changes in the course of normal brain aging are sexually dimorphic. Proc Natl Acad Sci U S A 2008; 105(40): 15605-10.

23. Rahman I, Gilmour PS, Jimenez LA, MacNee W. Oxidative stress and TNF-alpha induce histone acetylation and NF-kappaB/AP-1 activation in alveolar epithelial cells: potential mechanism in gene transcription in lung inflammation. Mol Cell Biochem, 2002; 234-235(1-2): 239-48.

24. Forbes MP, O’Neil A, Lane M, Agustini B, Myles N, Berk M. Major depressive disorder in older patients as an inflammatory disorder: Implications for the pharmacological management of geriatric depression. Drugs Aging 2021; 38(6):451-67.

25. Wang XM, Zhang YG, Li AL, et al. Relationship between levels of inflammatory cytokines in the peripheral blood and the severity of depression and anxiety in patients with Parkinson's disease. Eur Rev Med Pharmacol Sci 2016. 20(18): 3853-6. 